\title{
The effects of graded motor imagery and its components on phantom limb pain and disability in upper and lower limb amputees: a systematic review protocol
}

Katleho Limakatso ${ }^{1,2^{*}}$ D, Lieselotte Corten ${ }^{1}$ and Romy Parker ${ }^{1}$

\begin{abstract}
Background: Phantom limb pain (PLP) is characterized by the anatomical shifting of neighbouring somatosensory and motor areas into a deafferented cortical area of the brain contralateral to the amputated limb. It has been shown that maladaptive neuroplasticity is positively correlated to the perception of PLP in amputees. Recent studies support the use of graded motor imagery (GMI) and its component to alleviate the severity of PLP and disability. However, there is insufficient collective empirical evidence exploring the effectiveness of these treatment modalities in amputees with PLP. This systematic review will therefore explore the effects of GMI and its individual components on PLP and disability in upper and lower limb amputees.
\end{abstract}

Methods: We will utilize a customized search strategy to search PubMed, Cochrane Central register of Controlled Trials, MEDLINE, Embase, PsycINFO, PEDro, Scopus, CINAHL, LILACS, DARE, Africa-Wide Information and Web of Science. We will also look at clinicaltrials.gov (http://www.clinicaltrials.gov/), Pactr.gov (http://www.pactr.org/) and EU Clinical trials register (https://www.clinicaltrialsregister.eu/) for ongoing research. Two independent reviewers will screen articles for methodological validity. Thereafter, data from included studies will be extracted by two independent reviewers through a customized pre-set data extraction sheet. Studies with a comparable intervention and outcome measure will be pooled for meta-analysis. Studies with high heterogeneity will be analysed through random effects model. A narrative data analysis will be considered where there is insufficient data to perform a meta-analysis.

Discussion: Several studies investigating the effectiveness of GMI and its different components on PLP have drawn contrasting conclusions regarding the efficacy and applicability of GMl in clinical practice. This systematic review will therefore gather and critically appraise all relevant data, to generate a substantial conclusion and recommendations for clinical practice and research on this subject.

\section{Systematic review registration: PROSPERO CRD42016036471}

Keyword: Phantom limb pain, Disability, Graded motor imagery, Laterality recognition, Explicit motor imagery, Mirror visual feedback

Abbreviations: PLP, Phantom limb pain; GMI, Graded motor imagery; M1, Primary motor; S1, Somatosensory; PRISMA-P, Preferred Reporting Items of Systematic Reviews and Meta-Analyses Protocol; HRQoL, Health-related quality (Continued on next page)

\footnotetext{
* Correspondence: Imkmax001@myuct.ac.za

'Division of Physiotherapy, Department of Health and Rehabilitation

Sciences, University of Cape Town, Cape Town, South Africa

${ }^{2}$ Division of Physiotherapy, Department of Health and Rehabilitation

Sciences, Groote Schuur Hospital, University of Cape Town, F45 Old Main

Building, Cape Town, South Africa
} 
(Continued from previous page)

of life; PGIC, Patient global impressions of change; PEDro, Physiotherapy Evidence Database; CINAHL, Cumulative Index to Nursing and Allied Health Literature; DARE, Database of Abstracts of Reviews of Effects; Cl, Confidence interval;

MD, Mean difference; RR, Risk ratio; NNT, Number needed to treat; IMMPACT, Initiative on Methods, Measurement, and

Pain Assessment in Clinical Trials

\section{Background}

\section{Description of condition}

Amputation is the removal of a body extremity which is generally caused by severe trauma, circulatory disorders, neoplasm, deformities and infection of the limb. In case of gangrene, infection or neoplasm, amputation is carried out as a control strategy for pre-operative pain or a disease process in the affected limb; in some cases, however, amputation surgery is performed as a preventative procedure for the abovementioned complications [31]. Despite this attempt to alleviate patients' pain and disability, up to $80 \%$ of amputees report phantom limb pain (PLP) postamputation surgery [8]. PLP is defined as persistent painful sensations perceived in the missing portion of the amputated limb [7]. Previous research associates PLP with peripheral changes such as increased nociceptive input from the residual limb [39] and reduced near-surface blood-flow [34]. However, recent evidence suggests that PLP is a sensory output primarily driven by cortical changes in the brain [10]. Neuroimaging studies of patients with PLP revealed neuroplastic alterations of the somatotopic organization of the cortical and sub-cortical areas of the brain $[9,13]$. These changes are characterized by the anatomical shifting of neighbouring somatosensory [18] and motor [4] areas into a deafferented cortical area of the brain contralateral to the amputated limb. Furthermore, these neuroplastic changes are positively correlated to the severity of PLP $[15,17]$. These neuroplastic alterations can be reverted, with a correlation between the reversal of neuroplastic changes and pain relief in amputees with PLP $[1,11,20]$.

\section{Description of intervention}

Graded motor imagery (GMI) is a treatment strategy which has been shown to mitigate the severity of PLP and disability using a sequence of strategies including laterality recognition, explicit motor imagery and mirror visual feedback [22].

Laterality recognition, the ability to distinguish left from right, is dependent on the intact body schema in the brain and is important in the planning of movement [32]. This left/right judgement is inaccurate and delayed in amputees with PLP [25]. In this first phase of GMI, images representing the amputated limb are presented randomly on a computer screen. The patient is then instructed to match the side of the presented limbs by pressing either the left or right key. During this task, emphasis is put on accuracy and speed.

Laterality recognition is alternately known as implicit motor imagery, primarily because the patient is unconscious of mental movement processes involved to match the limb presented on the computer screen [5].

During the explicit motor imagery phase of GMI, the patient mentally moves the amputated limb to adopt a desirable posture presented on the computer screen [14].

The last strategy of GMI is mirror visual feedback, during which the amputated limb is concealed behind a mirror with the intact limb positioned comfortably in front of the mirror. This superimposes the ocular image of the intact limb on the phantom limb [28]. The patient is shown a picture of the unaffected limb in an easy to attain position. The patient then simultaneously moves the intact limb and the phantom limb (through imagination) to the presented position while observing the reflection of the intact limb in the mirror. This phase can commence with gross movements, progress to fine motor tasks and ultimately functional tasks.

\section{How the intervention might work}

GMI is consistent with sequential activation of cortical premotor and motor networks [23]. The GMI intervention is founded on the principle of graded increase in activity, similar to that implemented in physiotherapy treatment modalities [2]. This graded exposure to activity aims to promote cortical re-organization without triggering the protective pain response.

Laterality recognition/implicit motor imagery tasks activate premotor and supplementary motor areas, with an exception of the primary motor (M1) cortex [24]. Laterality recognition is therefore fundamental in preparation for subsequent phases of the GMI programme.

Explicit motor imagery activates the somatosensory (S1), premotor and M1 cortices contralateral to the phantom limb [17]. Activation of these areas is proposed to alleviate the perception of pain associated with imagination and observation of movement. This phase builds upon laterality recognition and serves as a foundation for the successive GMI phase.

Mirror visual feedback addresses changes in the S1 and M1 cortices [26]. In addition, it provides visual input to the brain, that movement is executed normally without inhibition [21]. The therapeutic effect associated with mirror visual feedback may be due to activation of mirror neurons 
in the brain hemisphere contralateral to the amputated limb [3]. These mirror neurons have been shown to fire during observation and execution of movement [16, 30].

\section{Importance of doing this review}

Several studies $[3,19,20,22,36,38]$, investigating the effectiveness of GMI and its different components on PLP, have drawn contrasting conclusions regarding the efficacy and applicability of GMI in clinical practice.

This systematic review will therefore gather and critically appraise all relevant data, to generate a substantial conclusion and recommendations for clinical practice and research on this subject.

It is important to note that Plumbe and associates have published a protocol with a similar topic. Despite this similarity, there is a significant distinction between the two protocols. In their review, Plumbe et al. [27] indicate that they will investigate the efficacy of GMI on chronic pain. However, this review will explore the effects of GMI and its components, specifically on PLP and disability. Bowering et al. [2] conducted a systematic review on a topic of interest. However, there is a need to update their review as there have been new studies since their publication.

\section{Objectives}

The purpose of this review is to explore the effects of GMI and its individual components on PLP and disability in upper and lower limb amputees.

\section{Methods}

The protocol was developed according to the Preferred Reporting Items of Systematic Reviews and Meta-Analyses Protocol (PRISMA-P) guidelines [33] and has been registered on PROSPERO database (Ref: CRD42016036471). The PRISMA-P checklist is included as an additional file (Additional file 1).

\section{Criteria for selecting studies for this review} Types of studies

This systematic review will consider published and nonpublished randomized controlled trials, quasi-experimental studies, randomized controlled cross-over trials and quasiexperimental cross-over studies published in English.

\section{Types of participants}

Participants older than 18 years of age with unilateral amputation of the upper or lower limb who received intervention for their PLP three or more months postamputation surgery will be included in this review. Studies with participants with pathology of the intact opposite limb will be excluded.

\section{Types of interventions}

GMI and its individual components (laterality recognition, explicit motor imagery and mirror visual feedback) will be compared to no treatment, conventional physiotherapy or other interventions. Studies investigating the efficacy of GMI plus additional treatment will also be included.

\section{Conventional physiotherapy includes:}

- Transcutaneous electrical nerve stimulation

- Muscle relaxation/massage

- Heat/cryotherapy

- Acupuncture

- Exercise

- Biofeedback

Other interventions include:

- Pharmacological interventions

- Psychotherapy

- Deep brain stimulation

- Motor cortex stimulation

- Spinal cord stimulation

\section{Types of outcome measures}

Primary outcome measures

- Self-reported PLP as assessed through a standardized pain scale post-intervention

- Pain-related disability as assessed through a standardized function scale post-treatment

Secondary outcome measures:

- Health-related quality of life (HRQoL) as assessed by a standardized scale

- Adverse effects

- Psychosocial function as assessed by a standardized scale

- Patient global impressions of change (PGIC) after 3 months or more

\section{Search strategy for identification of studies}

Electronic searches

We will utilize a customized search strategy (Appendix) to search the following electronic databases: PubMed, Cochrane Central register of Controlled Trials, MEDLINE (via Ebscohost), Embase, PsycINFO (via Ebscohost), Physiotherapy Evidence Database (PEDro), Scopus, Cumulative Index to Nursing and Allied Health Literature (CINAHL) (via Ebscohost), LILACS, Database of Abstracts of Reviews of effects in the Cochrane Library (DARE) Africa-Wide Information (via Ebscohost) and Web of Science. We will also look at clinicaltrials.gov (http://www.clinicaltrials.gov/), 
Pactr.gov (http://www.pactr.org/) and EU Clinical trials register (https://www.clinicaltrialsregister.eu/) for ongoing research.

\section{Search of other sources}

The reference lists of identified studies will be searched for relevant additional trials. Experts in this field will be contacted for further identification of published, unpublished and ongoing studies with potential for inclusion in this review.

\section{Data collection and analysis Selection of studies}

Databases will be searched by one reviewer to identify potential titles and abstracts. Two reviewers will independently screen these titles and abstracts for methodological validity. Full articles of relevant studies will be obtained and scrutinized by two independent reviewers to determine eligibility for inclusion in this review. Should there be a disagreement between reviewers, a consensus will be reached through discussion. However, should this fail, a third reviewer will be requested to take a decision.

\section{Data extraction and management}

Data from included studies will be extracted by two independent reviewers through a customized pre-set data extraction sheet. Extracted data will include the following: country of origin, study design (parallel/cross-over/cluster, randomisation, allocation concealment and blinding) and professional discipline of clinician delivering the intervention; setting, number of participants per group and points estimates; comorbidities, exclusion/inclusion criteria, participants' age and gender; type and side of amputation, adverse effects, pain condition and period (months) postamputation; assessment tools, type of treatment and control intervention received; duration of treatment (minutes), frequency of treatment per week, follow-up period (weeks) and number of patients lost to follow-up; baseline, postintervention and follow-up results on outcome measures and author conflict of interest statement. Data will be recorded into Review Manager 5 [37]. Disagreements concerning data extraction will be resolved through discussion. A third reviewer will be consulted where a consensus cannot be reached.

\section{Assessment of risk of bias}

Two independent reviewers will utilize the Cochrane method for risk of bias assessment. Included studies will be classified as low, high or unclear risk of bias. Any disagreements between reviewers will be resolved through discussion. A third reviewer will be consulted where a consensus cannot be reached. We will acknowledge and report on concerns of bias that can influence the outcome of this review, in particular selection bias, performance bias and publication bias

\section{Measures of treatment effect}

For studies with a comparable outcome measure, we will pool data for meta-analysis. Continuous data will be presented as $95 \%$ confidence interval (CI) and mean difference (MD). The risk ratio (RR) and $95 \% \mathrm{CI}$ will be calculated for dichotomous data. Furthermore, the number needed to treat (NNT) will be calculated. According to Initiative on Methods, Measurement, and Pain Assessment in Clinical Trials (IMMPACT), $\geq 15 \%$ pain reduction is minimally significant, $\geq 30 \%$ pain reduction is moderately significant and $\geq 50 \%$ is considerably significant [6].

\section{Unit of analysis issues}

For cross-over experimental design studies, only the first set of data will be considered for analysis. When trials have more than one intervention group, the control group will be divided in half. Each group will separately be included in the meta-analysis [29].

We will classify follow-up outcome measures by duration; short-term (up to 3 months post-treatment), medium-term (3-9 months post-treatment) and long-term (longer than 2 years post-treatment).

\section{Dealing with missing data}

In case of missing data, we will contact authors to provide further information. When authors fail to provide information within 2 months, we will use intention-to-treat analysis for extrapolated data. The impact of this will be reported in the discussion section of the systematic review.

\section{Assessment of heterogeneity}

Clinical heterogeneity will be addressed by pooling studies which investigate the same intervention and outcomes in amputees with PLP. Methodological heterogeneity will be examined visually, whereas statistical heterogeneity will be assessed through $I^{2}$ statistics. We will consider a cut-off score of $50 \%$.

\section{Assessment of reporting biases}

We will compare the methods section of the articles with the results section. If sufficient data is included, we will assess reporting bias by using a funnel plot [35].

\section{Data synthesis}

Studies with a comparable intervention and outcome measure will be pooled for meta-analysis using Review Manager 5 [37]. We will use the random effects model to analyse studies with high heterogeneity. We will 
consider narrative data analysis where there is insufficient data to perform a meta-analysis.

\section{Sensitivity analysis:}

If numerous trials satisfy the inclusion criteria for this systematic review, sensitivity analysis will be conducted to examine the possibility of excluding studies with a high risk of bias.

\section{Subgroup analysis}

A subgroup analysis depending on age, gender or type of amputee will be performed when applicable.

\section{Grading the certainty of evidence}

We will grade the certainty of the evidence for all outcome measures using the Grading of Recommendations Assessment, Development, and Evaluation working group methodology [12]

\section{Discussion}

Given the prevalence of amputees with PLP and disability, it is imperative to generate a substantial conclusion regarding the application of motor imagery techniques in clinical practice. The proposed systematic review will therefore explore the efficacy of GMI and its components as treatment modalities for PLP and disability. GMI is a cost-effective and non-invasive treatment with limited adverse effects and complications. As such, significant outcomes from this review will guide patients and clinicians in their treatment selection. Furthermore, these significant findings will reinforce the implementation of GMI in clinical practice, therefore making it accessible to low income upper and lower limb amputees with PLP and disability.

\section{Appendix}

Search strategy

1. (graded motor imagery OR motor imagery programme OR movement representation techniques)

2. (laterality recognition OR left right judge* $\mathrm{OR}$ implicit motor imagery)

3. (imagined movement OR explicit motor imagery OR mental imagery)

4. (mirror visual feedback OR mirror therap* OR mirror technique* OR mirror box therap*)

5. (phantom limb pain OR phantom pain)

6. (amput*)

7. 1 OR 2 OR 3 OR 4

8. 5 AND 6

9. 7 AND 8

\section{Additional file}

Additional file 1: PRISMA-P (Preferred Reporting Items for Systematic review and Meta-Analysis Protocols) 2015 checklist: recommended items to address in a systematic review protocol*.

\section{Acknowledgements}

The authors would like to thank the University of Cape Town, Health Sciences library for providing technical support.

Funding

$\mathrm{KL}$ is supported by the Oppenheimer Memorial Trust (OMT), South Africa.

Availability of data and materials

Not applicable.

Authors' contributions

$\mathrm{KL}$ drafted the protocol. RP and LC conceptualized and edited the protocol.

All authors read and approved the final manuscript.

Competing interests

The authors declare that they have no competing interests.

Consent for publication

Not applicable.

Ethics approval and consent to participate

Not applicable.

Received: 26 May 2016 Accepted: 22 August 2016

Published online: 01 September 2016

References

1. Birbaumer N, Lutzenberger W, Montoya P, Larbig W, Unertl K, Töpfner S, Grodd W, Taub E, Flor H. Effects of regional anesthesia on phantom limb pain are mirrored in changes in cortical reorganization. J Neurosci. 1997; 17(14):5503-8.

2. Bowering KJ, O'Connell NE, Tabor A, Catley MJ, Leake HB, Moseley GL, Stanton TR. The effects of graded motor imagery and its components on chronic pain: a systematic review and meta-analysis. J Pain. 2013;14(1):3-13.

3. Chan BL, Witt R, Charrow AP, Magee A, Howard R, Pasquina PF, Heilman KM, Tsao JW. Mirror therapy for phantom limb pain. N Engl J Med. 2007;357(21): 2206-7.

4. Chen R, Cohen LG, Hallett M. Nervous system reorganization following injury. Neuroscience. 2002;111(4):761-73.

5. Coslett HB, Medina J, Kliot D, Burkey AR. Mental motor imagery indexes pain: the hand laterality task. Eur J Pain. 2010;14(10):1007-13.

6. Dworkin RH, Turk DC, Wyrwich KW, Beaton D, Cleeland CS, Farrar JT, Haythornthwaite JA, Jensen MP, Kerns RD, Ader DN, Brandenburg N Interpreting the clinical importance of treatment outcomes in chronic pain clinical trials: IMMPACT recommendations. J Pain. 2008;9(2):105-21.

7. Ehde DM, Czerniecki JM, Smith DG, Campbell KM, Edwards WT, Jensen MP, Robinson LR. Chronic phantom sensations, phantom pain, residual limb pain, and other regional pain after lower limb amputation. Arch Phys Med Rehabil. 2000;81(8):1039-44.

8. Ephraim PL, Wegener ST, MacKenzie EJ, Dillingham TR, Pezzin LE. Phantom pain, residual limb pain, and back pain in amputees: results of a national survey. Arch Phys Med Rehabil. 2005;86(10):1910-9.

9. Flor H. Phantom-limb pain: characteristics, causes, and treatment. Lancet Neurol. 2002;1(3):182-9.

10. Flor H, Elbert T, Knecht S, Wienbruch C, Pantev C, Birbaumer N, Larbig W, Taub E. Phantom-limb pain as a perceptual correlate of cortical reorganization following arm amputation. Nature. 1995;375(6531):482-4.

11. Foell J, Bekrater-Bodmann R, Diers M, Flor H. Mirror therapy for phantom limb pain: brain changes and the role of body representation. European J Pain. 2014;18(5):729-39.

12. Guyatt GH, Oxman AD, Vist GE, Kunz R, Falck-Ytter Y, Alonso-Coello P, Schünemann HJ. GRADE: an emerging consensus on rating quality of evidence and strength of recommendations. BMJ. 2008 (Clinical research ed). 
13. Hamzei F, Liepert J, Dettmers C, Adler T, Kiebel S, Rijntjes M, Weiller C. Structural and functional cortical abnormalities after upper limb amputation during childhood. Neuroreport. 2001;12(5):957-62.

14. Johnson S, Hall J, Barnett SA, Draper M, Derbyshire G, Haynes L, Rooney C, Cameron H, Moseley GL, C Williams AC, McCabe C. Using graded motor imagery for complex regional pain syndrome in clinical practice: failure to improve pain. Eur J Pain. 2012;16(4):550-61.

15. Karl A, Birbaumer N, Lutzenberger W, Cohen LG, Flor H. Reorganization of motor and somatosensory cortex in upper extremity amputees with phantom limb pain. J Neurosci. 2001;21(10):3609-18.

16. Kilner JM, Lemon RN. What we know currently about mirror neurons. Curr Biol. 2013;23(23):1057-62.

17. Lotze M, Flor H, Grodd W, Larbig W, Birbaumer N. Phantom movements and pain. An fMRI study in upper limb amputees. Brain. 2001;124(11):2268-77.

18. Mackert BM, Sappok T, Grüsser S, Flor H, Curio G. The eloquence of silent cortex: analysis of afferent input to deafferented cortex in arm amputees. Neuroreport. 2003;14(3):409-12.

19. MacLachlan M, McDonald D, Waloch J. Mirror treatment of lower limb phantom pain: a case study. Disabil Rehabil. 2004;26(14-15):901-4.

20. Maclver K, Lloyd DM, Kelly S, Roberts N, Nurmikko T. Phantom limb pain, cortical reorganization and the therapeutic effect of mental imagery. Brain. 2008;131(8):2181-91

21. Matthys K, Smits M, Van der Geest JN, Van der Lugt A, Seurinck R, Stam HJ, Selles RW. Mirror-induced visual illusion of hand movements: a functional magnetic resonance imaging study. Arch Phys Med Rehabil. 2009;90(4):675-81.

22. Moseley GL. Graded motor imagery for pathologic pain: a randomized controlled trial. Neurology. 2006;67(12):2129-34.

23. Moseley GL. Graded motor imagery is effective for long-standing complex regional pain syndrome: a randomised controlled trial. Pain. 2004;108(1):192-8.

24. Moseley GL, Schweinhardt P, Wise R, Tracey I. Virtual, imagined and mirror movements - a novel approach to complex regional pain syndrome (CRPS1). Prague, Czech Republic: Proceedings of European Federation of IASP Chapters Triennial Conference; 2003.

25. Nico D, Daprati E, Rigal F, Parsons L, Sirigu A. Left and right hand recognition in upper limb amputees. Brain. 2004;127(1):120-32.

26. Nojima I, Mima T, Koganemaru S, Thabit MN, Fukuyama H, Kawamata T. Human motor plasticity induced by mirror visual feedback. J Neurosci. 2012; 32(4):1293-300.

27. Plumbe L, Peters S, Bennett S, Vicenzino B, Coppieters MW. Mirror therapy, graded motor imagery and virtual illusion for the management of chronic pain. Cochrane Database Syst Rev. 2016;(Issue 4). Art. No.: CD010329. doi:10. 1002/14651858.CD010329.pub2.

28. Ramachandran VS, Rogers-Ramachandran D. Synaesthesia in phantom limbs induced with mirrors. Proc R Soc Lond B Biol Sci. 1996;263(1369):377-86.

29. Ramsay C. 2003. Methodology in the Cochrane Effective Practice and Organisation of Care Group. How do you include trials with more than two groups into a single meta-analysis?. http://epoc.cochrane.org/sites/epoc. cochrane.org/files/uploads/FAQmultip. (Accessed 08 Jul 2016).

30. Rossi S, Tecchio F, Pasqualetti P, Ulivelli M, Pizzella V, Romani GL, Passero S, Battistini N, Rossini PM. Somatosensory processing during movement observation in humans. Clin Neurophysiol. 2002;113(1):16-24.

31. Schaper NC, Apelqvist J, Bakker K. The international consensus and practical guidelines on the management and prevention of the diabetic foot. Curr Diab Rep. 2003;3(6):475-9.

32. Schwoebel J, Coslett HB. Evidence for multiple, distinct representations of the human body. J Cogn Neurosci. 2005;17(4):543-53.

33. Shamseer L, Moher D, Clarke M, Ghersi D, Liberati A, Petticrew M, Shekelle P, Stewart LA. Preferred reporting items for systematic review and meta-analysis protocols (PRISMA-P) 2015: elaboration and explanation. BMJ. 2015;349:7647.

34. Sherman RA, Bruno GM. Concurrent variation of burning phantom limb and stump pain with near surface blood flow in the stump. Orthopedics. 1987; 10(10):1395-402

35. Sterne JAC, Egger M, Moher D. Addressing reporting biases. In: Higgins JPT, Chichester GS, editors. Cochrane Handbook for Systematic Reviews of Interventions. UK: Wiley; 2008. p. 297-334.

36. Sumitani M, Miyauchi S, McCabe CS, Shibata M, Maeda L, Saitoh Y, Tashiro T, Mashimo T. Mirror visual feedback alleviates deafferentation pain, depending on qualitative aspects of the pain: a preliminary report. Rheumatology. 2008; 47(7):1038-43
37. The Nordic Cochrane Centre, The Cochrane Collaboration. Review manager (RevMan). 5.1. Copenhagen: The Nordic Cochrane Centre, The Cochrane Collaboration; 2011.

38. Ülger Ö, Topuz S, Bayramlar K, Şener G, Erbahçeci F. Effectiveness of phantom exercises for phantom limb pain: a pilot study. J Rehabil Med. 2009;41(7):582-4.

39. Wall PD, Gutnick M. Ongoing activity in peripheral nerves: the physiology and pharmacology of impulses originating from a neuroma. Exp Neurol. 1974:43(3):580-93.

\section{Submit your next manuscript to BioMed Central and we will help you at every step:}

- We accept pre-submission inquiries

- Our selector tool helps you to find the most relevant journal

- We provide round the clock customer support

- Convenient online submission

- Thorough peer review

- Inclusion in PubMed and all major indexing services

- Maximum visibility for your research

Submit your manuscript at www.biomedcentral.com/submit
) Biomed Central 